\title{
MicroRNA-196a promotes non-small cell lung cancer cell proliferation and invasion through targeting HOXA5
}

Xiang-hua Liu ${ }^{1 \dagger}$, Kai-hua Lu ${ }^{2,3+}{ }^{+}$Ke-ming Wang ${ }^{4 \dagger}$, Ming Sun ${ }^{1}$, Er-bao Zhang ${ }^{1}$, Jin-song Yang ${ }^{5}$, Dan-dan Yin ${ }^{1}$, Zhi-li Liư ${ }^{4}$, Jing Zhou', Zhi-jun Liu', Wei De ${ }^{1 *}$ and Zhao-xia Wang ${ }^{4 *}$

\begin{abstract}
Background: MicroRNAs (miRNAs) are short, non-coding RNAs ( 22 nt) that play important roles in the pathogenesis of human diseases by negatively regulating gene expression. Although miR-196a has been implicated in several other cancers, its role in non-small cell lung cancer (NSCLC) is unknown. The aim of the present study was to examine the expression pattern of miR-196a in NSCLC and its clinical significance, as well as its biological role in tumor progression.

Methods: Expression of miR-196a was analyzed in 34 NSCLC tissues and five NSCLC cell lines by quantitative reverse-transcription polymerase chain reaction (qRT-PCR). The effect of DNA methylation on miR-196a expression was investigated by 5-aza-2-deoxy-cytidine treatment and bisulfite sequencing. The effect of miR-196a on proliferation was evaluated by MTT and colony formation assays, and cell migration and invasion were evaluated by transwell assays. Analysis of target protein expression was determined by western blotting. Luciferase reporter plasmids were constructed to confirm the action of miR-196a on downstream target genes, including HOXA5. Differences between the results were tested for significance using Student's t-test (two-tailed).

Results: miR-196a was highly expressed both in NSCLC samples and cell lines compared with their corresponding normal counterparts, and the expression of miR-196a may be affected by DNA demethylation. Higher expression of miR-196a in NSCLC tissues was associated with a higher clinical stage, and also correlated with NSCLC lymph-node metastasis. In vitro functional assays demonstrated that modulation of miR-196a expression affected NSCLC cell proliferation, migration and invasion. Our analysis showed that miR-196a suppressed the expression of HOXA5 both at the mRNA and protein levels, and luciferase assays confirmed that miR-196a directly bound to the 3'untranslated region of HOXA5. Knockdown of HOXA5 expression in A549 cells using RNAi was shown to promote NSCLC cell proliferation, migration and invasion. Finally, we observed an inverse correlation between HOXA5 and miR-196a expression in NSCLC tissues.
\end{abstract}

Conclusions: Our findings indicate that miR-196a is significantly up-regulated in NSCLC tissues, and regulates NSCLC cell proliferation, migration and invasion, partially via the down-regulation of HOXA5. Thus, miR-196a may represent a potential therapeutic target for NSCLC intervention.

Keywords: Non-small cell lung cancer, miR-196a, Proliferation, Invasion, HOXA5

\footnotetext{
* Correspondence: dewei@njmu.edu.cn; misswangzhaoxia@yahoo.com.cn

${ }^{\dagger}$ Equal contributors

${ }^{1}$ Department of Biochemistry and Molecular Biology, Nanjing Medical

University, Nanjing, People's Republic of China

${ }^{4}$ Department of Oncology, Second Affiliated Hospital, Nanjing Medical

University, Nanjing, People's Republic of China

Full list of author information is available at the end of the article
} 


\section{Background}

Non-small cell lung cancer (NSCLC) including adenocarcinoma and squamous cell carcinoma, is the predominant form of lung cancer, and accounts for the majority of cancer deaths worldwide [1]. Despite recent advances in clinical and experimental oncology, the prognosis of lung cancer is still unfavorable, with a 5-year overall survival rate of approximately $11 \%$ [2]. Thus, a detailed understanding of the mechanisms underlying NSCLC development and progression are essential for improving the diagnosis, prevention and treatment of this disease. Recently, accumulating evidence has shown that noncoding small RNAs may be involved in NSCLC pathogenesis, providing new insights into disease biology.

MicroRNAs (miRNAs) are 21-24 nucleotide, small, non-coding RNAs that regulate gene expression by base pairing with target mRNAs in the 3 '-untranslated region ( $3^{\prime}$-UTR), leading to mRNA cleavage or translational repression $[3,4]$. Dysregulation of miRNAs may lead to alterations in cellular differentiation, proliferation and apoptotic processes that are important in the development of cancer $[5,6]$. Indeed, deregulation of miRNAs is closely associated with tumor initiation, promotion and progression via the regulation of key oncogenes or tumor suppressors $[7,8]$. Thus, elucidating the biological consequences of miRNA dysregulation and identifying miRNA targets are critical for a complete understanding of miRNA pathways and their underlying molecular mechanisms.

The mature miR-196a is transcribed from two miR$196 a$ genes, miR-196a-1 and miR-196a-2. Recently, miRNA profiling studies indicate that miR-196a is overexpressed in several tumor tissues, including NSCLC [9-12]. Furthermore, an increasing number of reports indicate that miR-196a plays important roles in development, immunity and tumor pathogenesis via the targeting of specific genes [13-17]. In this study, we demonstrate that increased miR-196a expression is a characteristic molecular change in NSCLC and investigate the effect of increased miR-196a levels on the phenotypes of NSCLC cell lines. We also show that miR-196a may function as an oncogene by directly targeting HOXA5.

\section{Methods}

\section{Patient and tissue samples}

Paired NSCLC and adjacent non-tumor lung tissues were obtained from 34 consecutive patients who underwent primary surgical resection of NSCLC with informed consent between 2006 and 2007 at First Affiliated Hospital of Nanjing Medical University, China. Surgically laser capture micro-dissected NSCLC and adjacent normal tissues were immediately snap-frozen in liquid nitrogen and stored at $-80^{\circ} \mathrm{C}$ until total RNA was extracted. Tumor samples were at least $80 \%$ composed of viable-appearing tumor cells on histological assessment. The pathological stage, grade, and nodal status were appraised by an experienced pathologist. Clinicopathologic characteristics including tumor-nodemetastasis (TNM) staging had been collected. The study was approved by the Research Ethics Committee of Nanjing Medical University, China.

\section{Cell lines and culture conditions}

Four NSCLC adenocarcinomas cell lines (A549, SPC-A1, NCI-H1650, NCI-H1299), a NSCLC squamous carcinomas cell line (SK-MES-1), a normal human bronchial epithelial cell line (16HBE), and a human embryonic kidney cell line (HEK293T) were purchased from the Institute of Biochemistry and Cell Biology of the Chinese Academy of Sciences (Shanghai, China). Cells were cultured in RPMI 1640 or DMEM (GIBCO-BRL) medium supplemented with $10 \%$ fetal bovine serum (10\% FBS), $100 \mathrm{U} / \mathrm{ml}$ penicillin, and $100 \mathrm{mg} / \mathrm{ml}$ streptomycin (invitrogen) in humidified air at $37^{\circ} \mathrm{C}$ with $5 \% \mathrm{CO} 2$.

\section{RNA extraction and qRT-PCR analyses}

Total RNA was isolated with TRIzol reagent (Invitrogen, Carlsbad, CA, USA) according to the manufacturer's protocol. qRT-PCR assays were performed to detect miR-196a and HOXA5 expression using the PrimeScript RT reagent Kit and SYBR Premix Ex Taq (TaKaRa, Dalian, China) according to the manufacturer's instructions.

The relative level of miR-196a was determined by qRT-PCR using gene specific primers. U6 was validated as the normalizer, since which expression showed minimal variation in different cell lines and cancer specimens. The RT primers were designed as follows: miR-196a, 5' GTCAGAAGGAATGATGCA CAGCCAACAACA 3' and U6, 5' AACGCTTCACG AATTTGCGT 3'. The RT reaction was carried out under the following conditions: $42^{\circ} \mathrm{C}$ for $15 \mathrm{~min} ; 85^{\circ} \mathrm{C}$ for $5 \mathrm{sec}$; and then held on $4^{\circ} \mathrm{C}$. After the RT reaction, the complementary DNA products were diluted at $1: 100$, and $1 \mathrm{ul}$ of the diluted complementary DNA was used for subsequent qRT-PCR reactions.

The PCR primers for mature miR-196a or U6 were designed as follows: miR-196a sense, 5' CGTCAGAAGGAATGATGCACAG 3' and reverse, 5' ACCTGCGTAGGTAGTTTCATGT 3'; U6 sense, 5' CTCGCTTCGGCAGCACA 3' and reverse, 5' AACGCTTCACGAATTTGCGT 3'. The PCR reaction was conducted at $95^{\circ} \mathrm{C}$ for $30 \mathrm{~s}$ and followed by 40 cycles of $95^{\circ} \mathrm{C}$ for $5 \mathrm{~s}$ and $60^{\circ} \mathrm{C}$ for $34 \mathrm{~s}$ in the $\mathrm{ABI} 7500$ real-time PCR system (Applied Biosystems, Foster City, CA, USA). The qRT-PCR results were analyzed and expressed as relative miRNA expression of CT 
(threshold cycle) value, which was then converted to fold changes.

For analysis of HOXA5 mRNA expression, $100 \mathrm{ng}$ total RNA was reverse transcribed in a final volume of $10 \mu \mathrm{l}$ using random primers under standard conditions. HOXA5-specific primers were designed as follows: sense, 5' TCTCGTTGCCCTAATTCATCTTTT3' and reverse, 5' CATTCAGGACAAAGA GATGAACAGAA 3'. To verify integrity of HOXA5 expression, GAPDH gene was used as an internal control and the sequences of primers were as follows: sense, 5' GGGAGC CAAAAGGGTCAT 3' and reverse, 5' GAGTCCTTC CACG ATACCAA 3'. The relative levels of HOXA5 mRNA were calculated based on the difference between amplification of HOXA5 and GAPDH mRNA using the $2^{-\Delta} \mathrm{ct}$ method. All experiments were performed three times with three technical replicates.

\section{CpG island identification and methylation analysis}

Using the CpG Island Searcher Web tool (http://www. ebi.ac.uk/Tools/emboss/ cpgplot/index.html), a CpG island was identified upstream ( $-500 \mathrm{bp})$ of the region encoding miRNA-196a-1. For determination of the methylation status of the CpG island, genomic DNA prepared from $16 \mathrm{HBE}$ cells, was modified by sodium bisulfite (EZ DNA Methylation Kit, Zymo Research), followed by PCR using the sense primer 5' TTTTTTAG GATAGGAGGGGAT 3' and reverse, 5' TCTAAATCCT TAACCCC CTAAC 3', respectively. PCR-amplified product was transformed into E.coli DH5 $\alpha$ cells. Subsequently obtained plasmids were subjected to sequencing.

\section{Treatment of $16 \mathrm{HBE}$ cells with 5-aza-2-deoxy-cytidine (5-aza-CdR)}

$16 \mathrm{HBE}$ cells $\left(2.5 \times 10^{5}\right)$ were seeded into six-well culture plate on day 0 and exposed to 0,2 or $5 \mu \mathrm{M} 5$-aza-CdR (Sigma-Aldrich)for day 1 to day 3. Thereafter, the cells treated with 5 -aza-CdR were harvested on day 3 and used for detection of miR-196a expression or methylation analysis.

\section{Transfection of NCSCL cells}

All plasmid vectors (pCDNA/miR-196a and pCDNA/ miR-NC ) for transfection were extracted by DNA Midiprep or Midiprep kit (Qiagen, Hilden, Germany). Mature miR-196a mimics and miR-196a inhibitors (Anti-miR196a) were purchased from Sigma-Aldrich and Applied Biosystems, AB, Ambion, respectively. The appropriate negative controls (miR-NC or anti-miR-NC) were synthesized by GenePharma, Shanghai, China. HOXA5siRNA named si-HOXA5 and non-specific control siRNA (si-NC) were purchased from genechem, Shanghai, China. The pCDNA/miR-196a, mature miR-196a mimics or si-HOXA5 was transfected into cultured
A549 cells respectively, and miR-196a inhibitors were transfected into cultured SPC-A1 cells. A549 or SPC-A1 cells were grown on six-well plate to confluence and transfected using Lipofectamine2000 (Invitrogen) according to the manufacturer's instructions. Fourtyeight hours after transfection, cells were harvested for qRT-PCR analyses or Western blot.

\section{Cell proliferation assays}

Cell proliferation was monitored using Cell Proliferation Reagent Kit I (MTT) (Roche Applied Science). MiR196a inhibitors transfected SPC-A1 cells (3000/well), and pCDNA/miR-196a or si-HOXA5 transfected A549 cells (2000/well) were allowed to grow in 96-well plates. Cell proliferation was documented every $24 \mathrm{~h}$ following the manufacturer's protocol. All experiments were performed in quadruplicate. For the colony formation assay, a total of 500 miR-196a inhibitors transfected SPC-A1, pCDNA/miR-196a or si-HOXA5 transfected A549 cells were placed in a fresh six-well plate and maintained in media containing $10 \%$ FBS, replacing the medium every 4 days. After 14 days, cells were fixed with methanol and stained with $0.1 \%$ crystal violet (sigma). Visible colonies were manually counted. Triplicate wells were measured in each treatment group.

\section{Flow-cytometric analysis of apoptosis}

SPC-A1 cells transiently transfected with miR-196a inhibitors or anti-miR-NC, were harvested at $48 \mathrm{~h}$ after transfection by trypsinization. After the double staining with FITC-Annexin V and Propidium iodide (PI), the cells were analyzed with a flow cytometry $\left(\right.$ FACScan ${ }^{\circledR}$; BD Biosciences) equipped with a CellQuest software (BD Biosciences) [18]. Cells were discriminated into viable cells, dead cells, early apoptotic cells, and apoptotic cells, and then the relative ratio of early apoptotic cells were compared to control from each experiment. All of the samples were assayed in triplicate.

\section{Hoechst staining assay}

SPC-A1 cells transfected with miR-196a inhibitors were cultured in six-well plates, and were incubated with Hoechst 33342 solution $(50 \mathrm{ng} / \mathrm{ml}$, Sigma, St Louis, MO, USA) for $10 \mathrm{~min}$ at room temperature. Cells were then washed twice with PBS and changes in nuclear morphology were detected by fluorescence microscopy using a filter for Hoechst $33342(365 \mathrm{~nm})$. For quantification of Hoechst 33342 staining, the percentage of Hoechst -positive nuclei per optical field (at least 50 fields) was counted in three independent experiments.

\section{Cell migration and invasion assays}

For the migration assays, $24 \mathrm{~h}$ after transfection, $5 \times 10^{4}$ cells in serum-free media were placed into the upper 
chamber of an insert (8- $\mu \mathrm{m}$ pore size, millepore). For the invasion assays, $1 \times 10^{5}$ cells in serum-free media were placed into the upper chamber of an insert coated with Matrigel(Sigma-Aldrich, USA). Media containing $10 \%$ FBS were added to the lower chamber. After several hours of incubation, the cells remaining on the upper membrane were removed with cotton wool, whereas the cells that had migrated or invaded through the membrane were stained with methanol and $0.1 \%$ crystal violet, imaged, and counted using an IX71 inverted microscope (Olympus, Tokyo, Japan). Experiments were independently repeated three times.

\section{Bioinformatics methods}

The miRNA targets predicted by computer-aided algorithms were obtained from pictar (http://pictar.mdc-berlin. de/cgi-bin/new_PicTar_vertebrate.cgi.), targetscan (http:// www.targetscan.org) and mirbase targets (http://microrna. sanger.ac.uk/cgi-bin/targets/v5/search.pl).

\section{Plasmid constructs and luciferase assay}

To construct a luciferase reporter vector, HOXA5 3'UTR fragment containing putative binding sites for miR196a was amplified by PCR using the following primers: sense-5'-GATGTTTTAACTTATTTATATGAAG-3' and reverse-5'-CAA ATATTGTCCAAGTCTGGCTGTT-3'. The PCR product was subcloned downstream of the luciferase gene in the pLUC Luciferase vector (Ambion, Inc., Austin, TX, USA) and named HOXA5-3'-UTR-WT. Sitedirected mutagenesis of the miR-196a target site in the HOXA5-3'-UTR was performed using the Quick-change mutagenesis kit (Stratagene, Heidelberg, Germany) and named HOXA5 -3'-UTR-Mut, in which 3'-UTR-WT was used as a template. For the mutated construct, the miR196a target site 5'- GATATTTTTATTCAAA CTACCTA -3' was substituted with a 5'-GATATTTTTATTCTTT GATGGAA -3' fragment.

Human HEK293T cells grown in a 48-well plate were co-transfected with $200 \mathrm{ng}$ of either mock pCDNA/ miR-NC or pCDNA/miR-196a, 10 ng of firefly luciferase reporter comprising wild type or mutant 3'UTR of HOXA5 gene, and 2 ng of pRL-TK (Promega, Madison, WI, USA) using Lipofectamie 2000 ( Invitrogen, USA). Cells were harvested $48 \mathrm{~h}$ after transfection for luciferase assay using a luciferase assay kit (Promega) according to the manufacturer's protocol. Each experiment was repeated triplicates.

\section{Western blotting assay}

Cells were lysed using mammalian protein extraction reagent RIPA (Beyotime) supplemented with protease inhibitors cocktail (Roche) and PMSF (Roche). Protein concentration was measured with the Bio-Rad protein assay kit. $50 \mu \mathrm{g}$ protein extractions were separated by
$12 \%$ SDS-polyacrylamide gel electrophoresis (SDSPAGE), then transferred to $0.22 \mu \mathrm{m}$ NC membranes (Sigma)and incubated with specific antibodies. Autoradiograms were quantified by densitometry (Quantity One software; Bio-Rad). GAPDH was used as control. GAPDH antibody and goat anti-HOXA5 (1:200) were purchased from sigma and Santa Cruz Biotechnology (Santa Cruz, CA, USA), respectively.

\section{Statistical analysis}

Student's t-test (two-tailed), One-way ANOVA and Mann-Whitney test were performed to analyze the data using SPSS 16.0 software. P values less than 0.05 were considered statistically significant.

\section{Results}

miR-196a expression is up-regulated in human NSCLC tissues

The level of miR-196a was detected in 34 NSCLC samples and adjacent, histologically normal tissues by qRTPCR, and normalized to U6. miR-196a expression was significantly up-regulated in cancerous tissues (median ratio of 22.9-fold, $\mathrm{p}<0.01$ ) compared with corresponding normal tissues. Furthermore, analysis of the correlation of miR-196a expression with clinical pathological features of NSCLC patients, revealed a significant association between miR-196a up-regulation and advanced pathological stage (I/II, $n=20)$ vs (III/IV, $n=14)$ and NSCLC lymph-node metastasis (Figure 1A). We next performed qRT-PCR analysis to examine the expression of miR-196a in five human NSCLC cell lines, including both adenocarcinoma and squamous carcinoma subtypes. Of these, four cell lines (SPC-A1, NCI-H1650, NCI-H1299 and SK-MES-1) expressed higher levels of miR-196a compared with the normal, bronchial epithelial cell line, 16HBE, while A549 cells expressed relatively low endogenous levels of miR-196a (Figure 1B). These results indicate that over-expression of miR-196a may play an important role in NSCLC progression and development.

\section{Effect of DNA methylation on miR-196a expression}

Since the expression of miR-196a was frequently upregulated in NSCLC, we investigated whether this might be mediated by epigenetic mechanisms, including DNA demethylation. We hypothesized that epigenetic modification of CpG-rich regions within the regulatory regions of miR-196a may be involved in aberrant transcriptional activation. Bioinformatic analysis identified a canonical CpG island in the promoter region of the miRNA-196a1 loci (Figure 2A); however, no canonical CpG island was found in the promoter region of the miRNA-196a-2 loci (data not shown). Following treatment of 16HBE cells with DNA demethylating agent (5-aza-CdR), the 


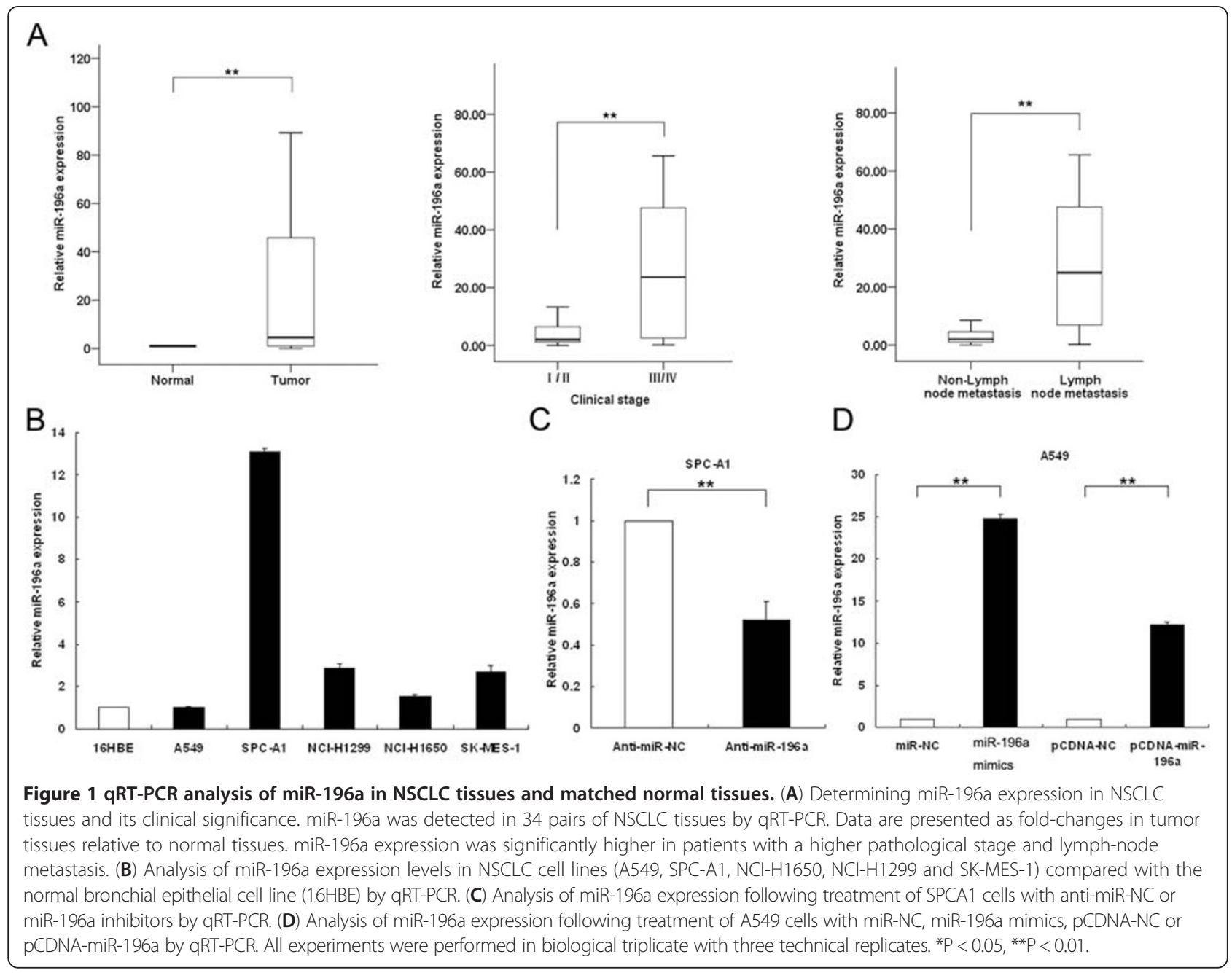

expression of miR-196a was determined by qRT-PCR (Figure 2B) and CpG island methylation was assessed by bisulfite sequencing (Figure $2 \mathrm{C}$ ). We found that miR196a expression was significantly increased 4.4- or 5.1fold in 5-aza-CdR treated cells compared with control, and the frequency of methylation was decreased from $78.2 \%$ to $67 \%$. These results indicate that up-regulation of miR-196a in NSCLC cells may be affected by DNA demethylation.

\section{Manipulation of miR-196a levels in NSCLC cells}

To selectively down-regulate or over-express miR-196a, miR-196a inhibitors or mature miR-196a mimics and their corresponding negative controls (anti-miR-NC or miR-NC, respectively) were transiently transfected into SPC-A1 or A549 cells. In addition, to stably sustain the expression of miR-196a in A549 cells, cells were transfected with pCDNA/miR-196a vector or pCDNA/ miR-NC empty vector control. qRT-PCR analysis of miR-196a levels was performed $48 \mathrm{~h}$ post-transfection, and revealed that miR-196a expression was reduced 0.52-fold following transfection with miR-196a inhibitors and increased 24.7-fold after transfection with miR-196a mimics. Expression of miR-196a was induced 12.2-fold in stably transfected A549/miR-196a cells, compared with control (Figure $1 \mathrm{C}$ and 1D).

\section{Effect of miR-196a on cell proliferation and apoptosis}

To assess the biological role of miR-196a in NSCLC, we investigated the effect of targeted knockdown or overexpression of miR-196a on cell proliferation and apoptosis. MTT assay revealed that cell growth was significantly impaired in SPC-A1 cells transfected with miR-196a inhibitors, while proliferation of A549 cells was increased in pCDNA/miR-196a transfected cells compared with controls (Anti-miR-NC or pCDNA/miR-NC, respectively) (Figure 3A and 3B). Similarly, the results of colony-formation assays revealed that clonogenic survival was decreased following inhibition of miR-196a in SPC-A1 cells, and enhanced in pCDNA/miR-196a transfected A549 cells (Figure 3C and 3D). 
A
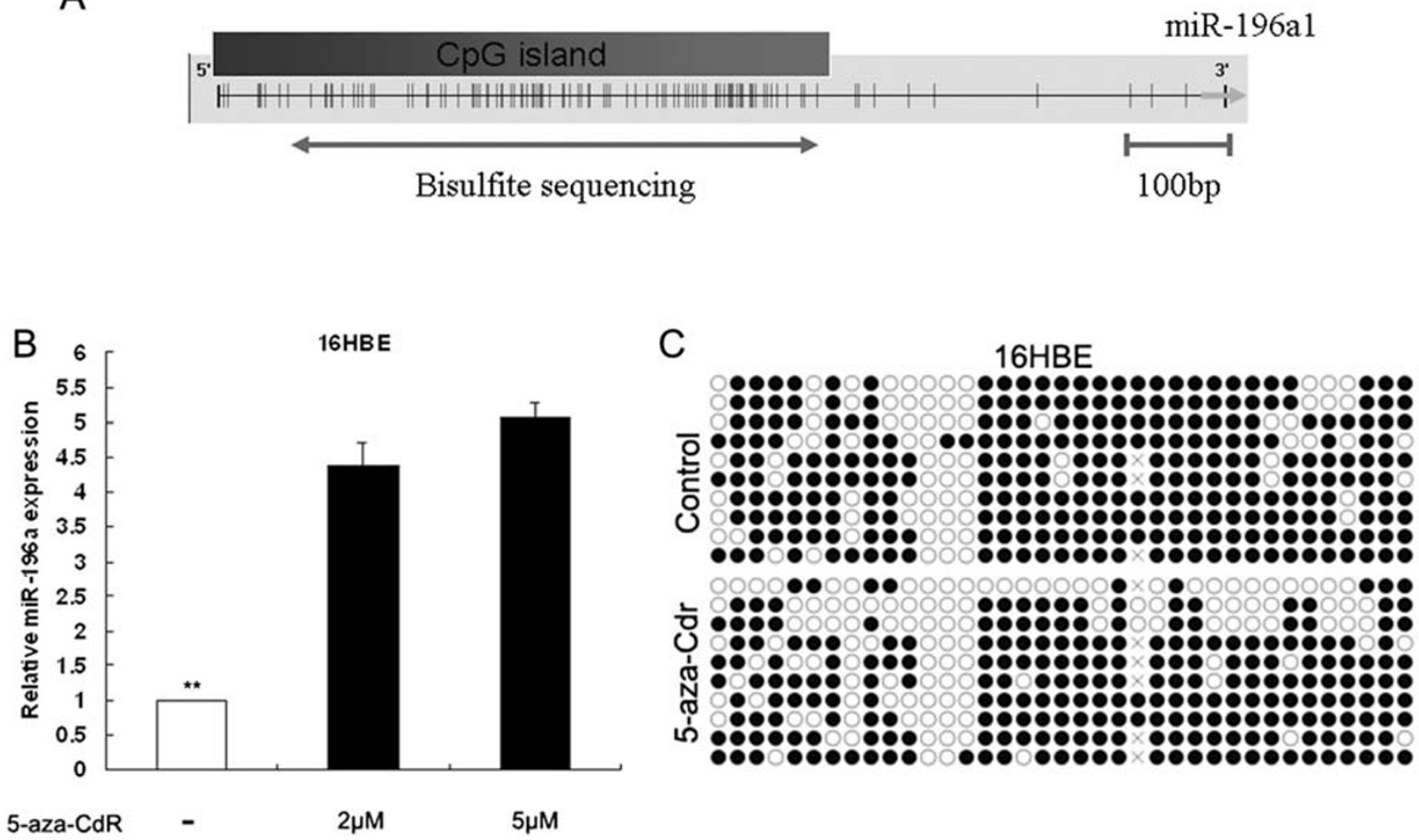

Figure 2 Analysis of the correlation between methylation status and expression of miR-196a. (A) Map of the CpG island position of miR-196a-1. Vertical ticks mark CpG sites. (B) The level of miR-196a expression in 16HBE cells after treatment of 5-aza-dC (0, 2, $5 \mu \mathrm{M})$. (C) The methylation status of the $\mathrm{CpG}$ island of miR-196a-1 was assessed by bisulfite sequencing before and after 5-aza-dC treatment in $16 \mathrm{HBE}$ cells. Open and filled squares denote unmethylated and methylated CpG sites, respectively. Each row represents a single clone. ${ }^{*} \mathrm{P}<0.05$; ${ }^{* *} \mathrm{P}<0.01$.

To determine whether apoptosis was a contributing factor to cell growth inhibition, we performed Hochest staining and flow-cytometric analysis of SPC-A1 cells after transfection with miR-196a inhibitors. Alteration of miR-196a expression had no significant effect on cell apoptosis compared with control cells (data not shown). Taken together, these results indicate that inhibition of miR-196a suppresses cell growth, but is not associated with induction of apoptosis.

\section{miR-196a promotes migration and invasion of NSCLC} cells

Cell invasion is a significant aspect of cancer progression, and involves the migration of tumor cells into contiguous tissues and the dissolution of extracellular matrix proteins. To investigate whether miR-196a had a direct functional role in facilitating NSCLC cell migration and invasion, we evaluated cancer cell invasion through Matrigel and migration through a transwell. As shown in Figure 4A, inhibition of miR-196a impeded the migration of SPC-A 1 cells by approximately $64 \%$ compared with control. Similarly, invasion of SPC-A1 cells was also reduced 59\% following inhibition of miR-196a. Conversely, transfection of A549 cells with miR-196a mimics promoted cell migration and invasion ability $\sim 2.5$-fold (Figure 4B). These data indicate that miR-196a is an onco-miRNA that can promote the migratory and invasive phenotype of NSCLC cells.

\section{HOXA5 is a direct target of miR-196a}

To explore the molecular mechanism by which miR196a contributes to the proliferation and invasion of NSCLC cells, we searched for potential targets as predicted by commonly cited programs such as TargetScan, PicTar and miRanda. Two candidate genes, HOXA5 and FOXO1, were selected for further analysis, owing to their relatively high prediction score and their complementary structure with miRNA-196a (Figure 5A). To verify whether these genes were direct targets of miR-196a, the wild-type 3' untranslated region (UTR) of HOXA5 and FOXO1 were fused directly downstream of the firefly luciferase gene (pLuc). pCDNA/miR-196a was then cotransfected with various luciferase 3' UTR constructs into HEK293T cells. MiR-196a significantly inhibited luciferase activity $(\sim 5 \%)$ of the wild-type pLuc-HOXA5 3'UTR reporter.

To further confirm that miR-196a-mediated reduction of luciferase activity from the pLuc-HOXA5 3'-UTR 


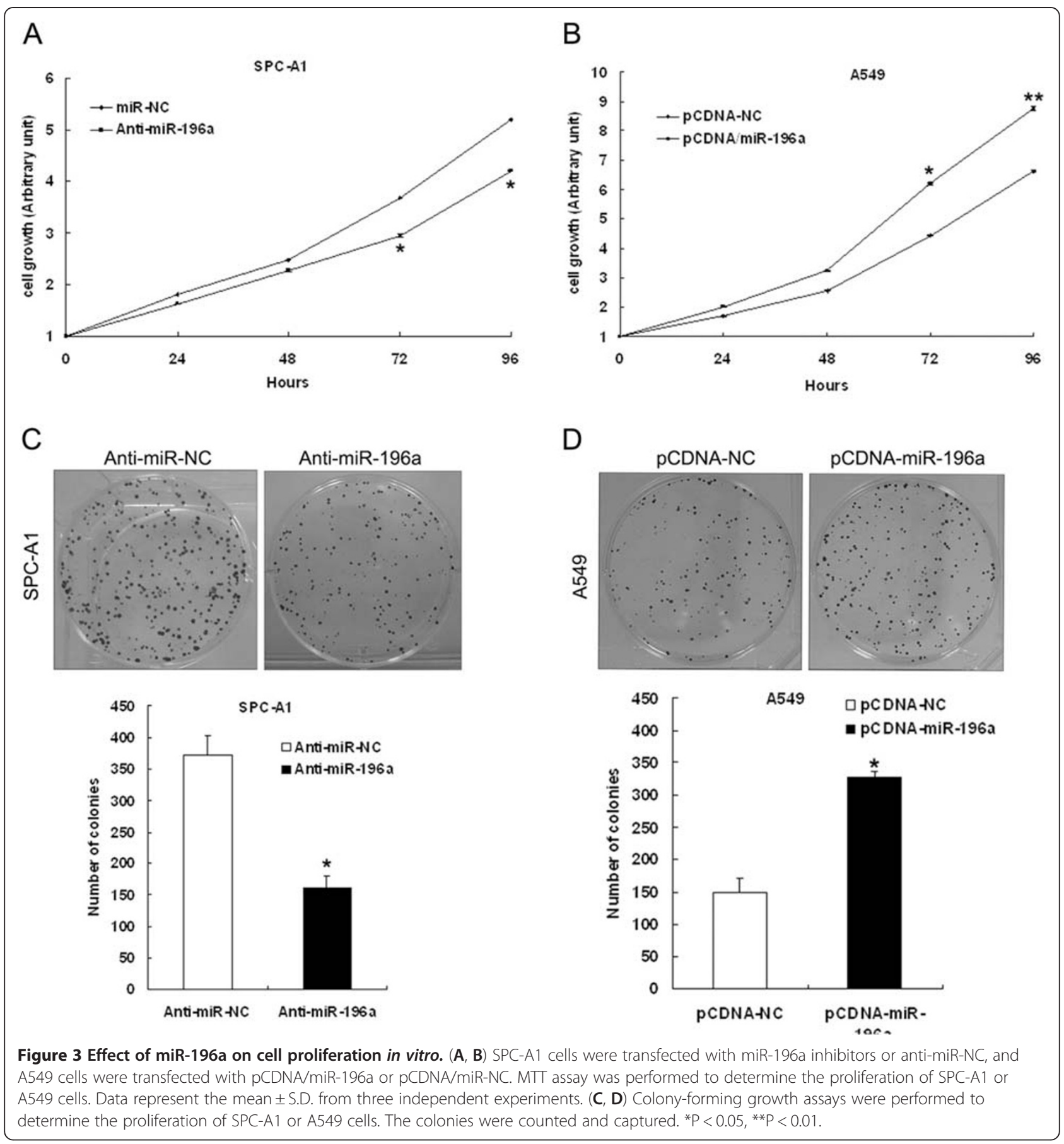

vector was due to direct interaction between miR-196a and its putative binding site, we mutated the miR-196a binding site by site-directed mutagenesis, resulting in pLuc-HOXA5-3'-UTR-Mut. As expected, suppression of luciferase activity was completely abolished in this mutant construct compared with wild-type vector (Figure 5B).

We next investigated whether miR-196a could regulate HOXA5 at both mRNA and protein levels. miR- 196a inhibitors or mimics were transfected into NSCLC cell lines, and the levels of HOXA5 mRNA and protein were monitored (Figure 5C and 5D). qRTPCR analysis revealed that inhibition of miR-196a in SPC-A1 cells led to increased expression of endogenous HOXA5 mRNA compared with control. Additionally, western blot analysis showed that HOXA5 protein expression was clearly up-regulated following transfection of SPC-A1 cells with miR-196a inhibitors. In 


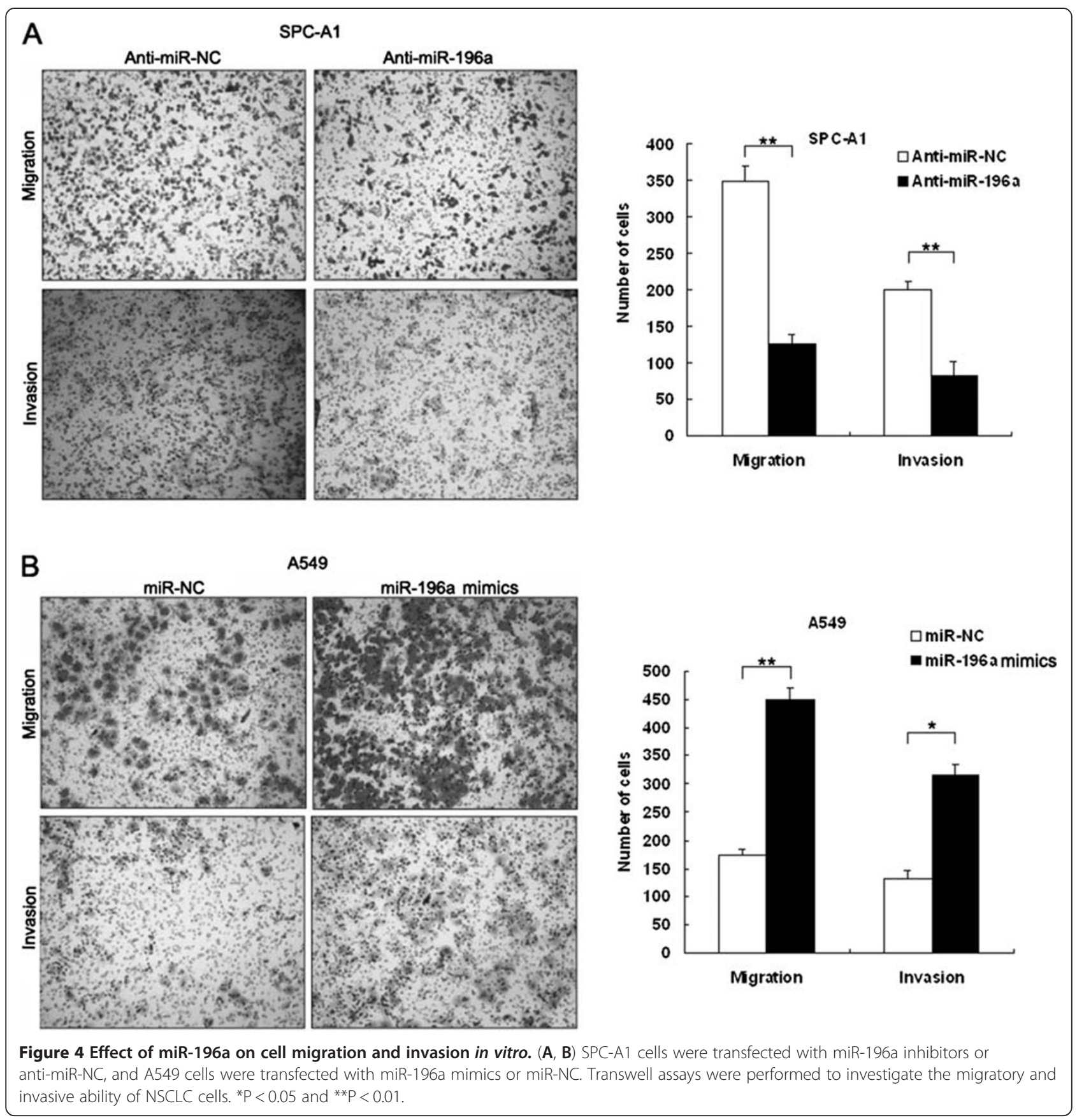

addition, enforced expression of miR-196a in A549 cells triggered a significant silencing effect on endogenous HOXA5 expression, both at the mRNA and protein level.

Effect of HOXA5 on NSCLC cells proliferation and invasion To determine whether HOXA5 could also inhibit NSCLC cell proliferation, migration and invasion, we performed targeted knockdown of HOXA5 expression using RNAi in A549 cells. The expression levels of HOXA5 mRNA and protein in A549/si-HOXA5 cells were significantly decreased compared with si-NC transfected cells (Figure 6A). Transwell migration assays revealed that inhibition of HOXA5 promoted cell migration and invasion (Figure 6B). Next, we performed MTT and colony formation assays to investigate cell growth and clonogenicity. A549 cells transfected with si-HOXA5 displayed a significantly enhanced growth ability compared with cells transfected with si-NC (Figure 6C, D). These data indicate that down-regulation of HOXA5 expression promotes NSCLC cell proliferation, migration and invasion, 


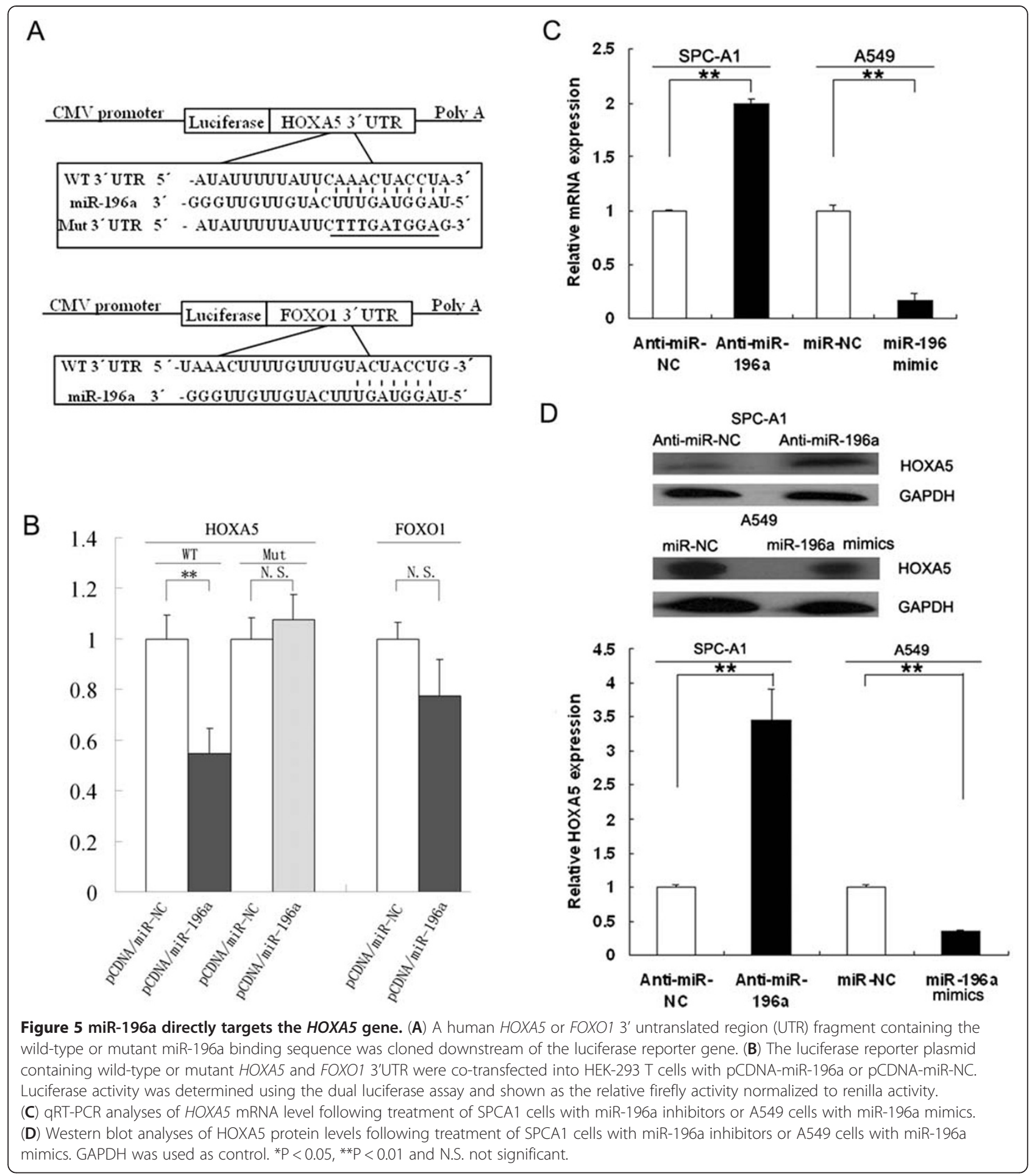

phenocopying the over-expression of miR-196a in A549 cells.

\section{Downregulation of HOXA5 is inversely correlated with} miR-196a expression in NSCLC

As miR-196a was over-expressed in NSCLC and targeted HOXA5 by binding to its 3 'UTR, we next determined whether HOXA5 expression was negatively associated with miR-196a levels in primary NSCLC patient tissues. Analysis of HOXA5 expression level in 34 NSCLC tissues and corresponding normal tissues by qRT-PCR revealed that $H O X A 5$ was significantly down-regulated in NSCLC (Figure 6E). Furthermore, bivariate correlation analysis revealed that low expression of HOXA5 


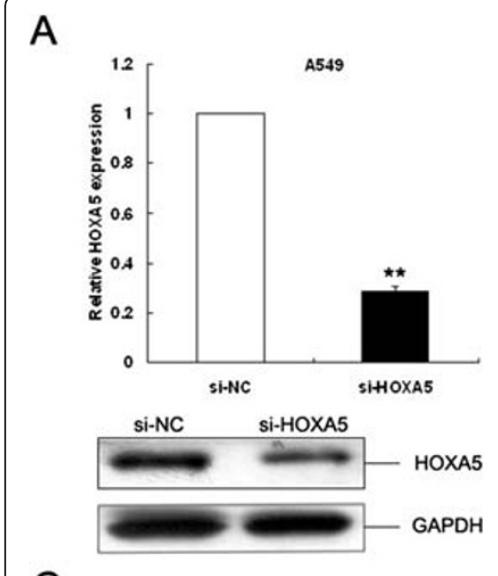

C

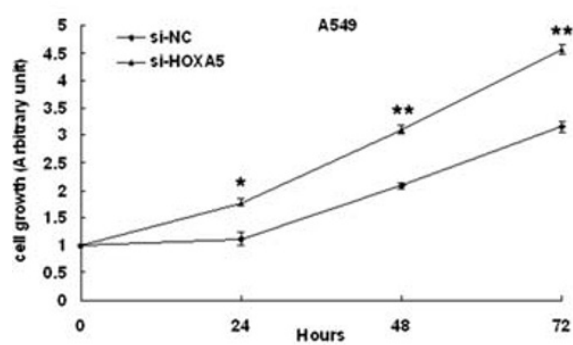

E

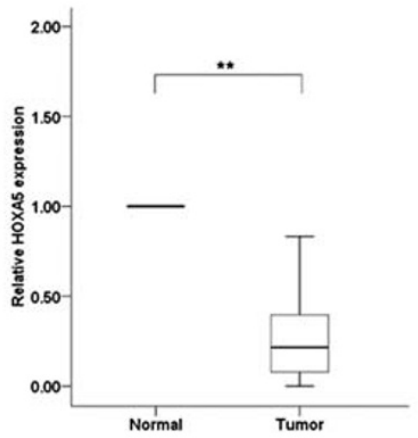

B
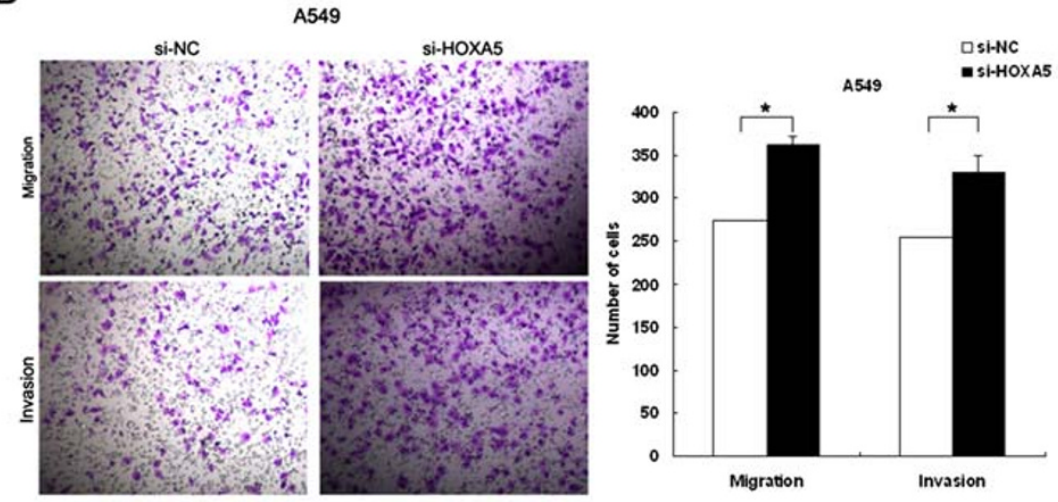

D

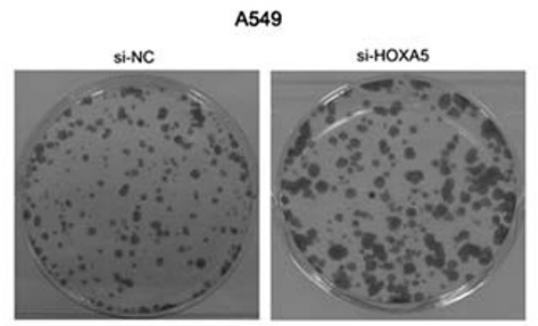

$\mathrm{F}$
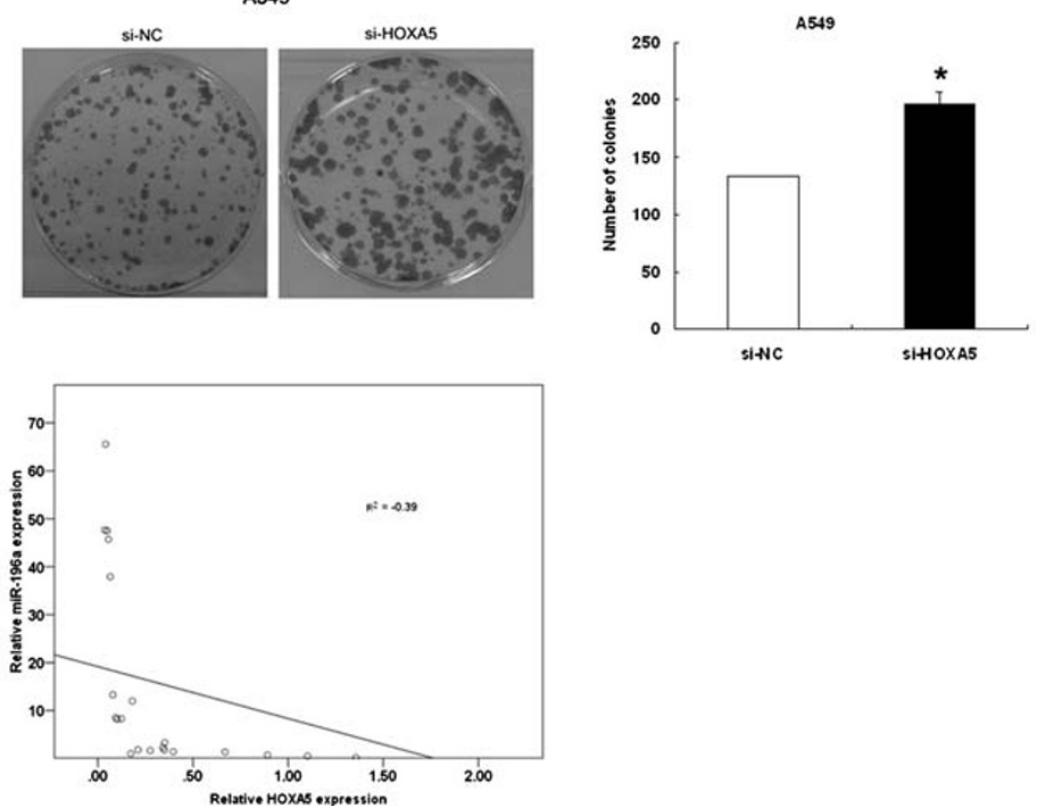

Figure 6 Effect of HOXA5 on NSCLC cell proliferation, migration and invasion. (A) A549 cells were transfected with si-HOXA5 or si-NC, and HOXA5 mRNA and protein levels were assessed by qRT-PCR and western blot. (B) Transwell assays were performed to investigate the migratory and invasive ability of NSCLC cells. (C, D) MTT assay and colony-forming growth assays were performed to determine the proliferation of si-NC or si-HOXA5 A549 cells. (E) The level of HOXA5 mRNA in NSCLC tissues was analyzed by qRT-PCR. (F) Analysis of the relationship between miR-196a expression and HOXA5 mRNA levels. ${ }^{* P}<0.05,{ }^{*} \mathrm{P}<0.01$.

was more likely correlated with high levels of miR-196a (Figure 6F), suggesting that the downregulation of HOXA5 may be due to enhanced miR-196a expression in NSCLC.

\section{Discussion}

The aberrant expression of miR-196a is a frequent event in various cancers, suggesting that miR-196a may play an important role in tumorigenesis and tumor progression. Indeed, several key oncogenic functions have been attributed to miR-196a in the context of tumorigenesis. In esophageal cancer, miR-196a over-expression promotes cell proliferation, anchorage-independent growth and suppresses apoptosis by directly regulating ANXA1 [16]. In colorectal cancer, high levels of miR-196a were observed to activate the Akt signaling pathway, promote cancer cell detachment, migration, invasion and chemosensitivity, and increase the development of lung metastases in mice [10,17]. In previous studies from our group, we reported that aberrant over-expression of miR-196a and the resultant down-regulation of its target p27kip1, contributes to gastric carcinogenesis [11]. We also analyzed miRNA expression profiles in NSCLC patient tissues by miRNA microarray, and identified miR$196 \mathrm{a}$ as the most highly up-regulated miRNA compared with corresponding normal tissues [12]. Inspired by the 
above observation, we investigated the biological role of miR-196a and explored the molecular mechanisms by which miR-196a modulates the behavior of NSCLC cells.

In this study, we first examined the expression of miR196a in 34 paired normal/tumor tissues from NSCLC patients, and then investigated the clinical implications. Consistent with our previous microarray analysis, miR196a was dramatically upregulated in NSCLC tissues. Specifically, miR-196a expression was significantly higher at later stages of NSCLC development or in specimens displaying more extensive metastasis compared with their normal counterparts. This suggests that high expression of miR-196a may be involved in NSCLC carcinogenesis. In addition, we demonstrate that miR-196a expression is elevated in NSCLC cell lines compared with the 16HBE normal human bronchial epithelial cell line, with the exception of A549 cells. We also show that DNA demethylation may underlie the aberrant expression of miR-196a in NSCLC, and these findings are currently under further investigation in our laboratory.

To assess the role of miR-196a in NSCLC, we investigated the gain-or-loss of function effects of miR-196a on various aspects of NSCLC biology. First, we demonstrated that targeted knock-down of miR-196a expression in SPC-A1 cells led to significant inhibition of cell proliferation, migration and invasion. Conversely, introducing miR-196a into A549 cells, which express relatively low levels of endogenous miR-196a, induced corresponding malignant tumor cell behaviors. However, unlike previous descriptions in breast cancer, alteration of miR-196a expression did not impact apoptosis in NSCLC cells. Therefore, the functional role of miR-196a may be tissue- and cell type-specific, and the detailed mechanisms of miR-196a and its targets are worthy of further study.

Identification of putative miRNA targets is important for a complete understanding of the specific functions of miRNAs. In this study, we identify HOXA5 as a direct target of miR-196a, and demonstrate that upregulation of miR-196a significantly reduces HOXA5 expression at both transcriptional and protein level in NSCLC cells.

Mammals have four $H O X$ gene clusters ( $H O X A-D)$, which act as master regulators that specify body patterning during embryonic development. As transcription factors, HOX proteins control a battery of target genes encoding cellular functions required for cell identity, cell growth and differentiation, as well as cell-cell and cellmatrix interactions [19-22]. Deregulation of HOX genes in cancer is now well established, although in general rather less is known about their function [23-28]. The homeobox protein, HOXA5, has been shown to participate in the developmental regulation of the lung. Mandeville et al. observed impaired postnatal lung development in $\mathrm{HOXA5}^{-/-}$mice, indicating that HOXA5 has a critical role in lung ontogeny, and implying its involvement in lung maturation and function [29]. Similarly, Packer et al. reported that HOXA5 is likely to be involved in development and patterning of the mouse lung [30]. Moreover, dysregulation of HOXA5 expression has been associated with lung tumorigenesis and other diseases in humans [26,31]. In breast cancer, down-regulation of HOXA5 may impact $p 53$ gene expression, contributing to the oncogenic process $[32,33]$. Additionally, induction of HOXA5 is important for retinoic acid (RA)-mediated apoptosis and cellular growth inhibition acting directly downstream of RAR $\beta$, and plays an important role in RA-mediated anti-cancer activity $[34,35]$.

Currently, the role of HOXA5 in NSCLC has not been well established. Our results reveal that inhibition of HOXA5 expression in A549 cells significantly promotes cell proliferation, migration and invasion, consistent with the results of ectopic miR-196a expression in the same cells. Furthermore, our observation of a correlation between elevated miR-196a levels and decreased HOXA5 levels in NSCLC tissues, indicates that down-regulation of HOXA5 may be a mechanism by which miR-196a exerts its oncogenic functions.

While our study provides critical insight into NSCLC pathogenesis, the existence of several limitations should be noted. First, as the number of tissue samples in this study was limited, further investigation of a larger patient cohort is essential to confirm the clinical significance of miR-196a. Second, to further understand the biological role of miR-196a in regulating NSCLC development and progression, a series of in vivo studies using xenograft models are required. Third, many studies have clearly indicated that one miRNA is capable of controlling multiple genes. Therefore, the observed miR-196a-mediated inhibition of cell growth and invasion is likely due to simultaneous targeting of multiple targets in NSCLC. Further studies of miR-196a will undoubtedly enhance our knowledge of how miR-196a functions in regulating NSCLC cell growth and metastasis.

\section{Conclusions}

In summary, we demonstrate that miR-196a overexpression is a common event underlying NSCLC, and may function as an oncogene by directly targeting HOXA5. We show, possibly for the first time, that the miR-196a/HOXA5 axis regulates the proliferation and invasion of NSCLC cells. These findings may help us to better understand the pathogenesis and development of NSCLC and facilitate the development of miRNAdirected diagnostics and therapeutics against this deadly disease. 


\section{Competing interests}

The authors declare that they have no competing interests.

\section{Authors' contributions}

$\mathrm{XHL}, E B Z$, and MS were involved in the conception and design of the study. $\mathrm{KHL}, \mathrm{KMW}, \mathrm{XHL}, \mathrm{EBZ}, \mathrm{MS}$ and DDY were involved in the provision of study material and patients. JSY, ZLL, JZ and ZJL performed the data analysis and interpretation. $X H L$ wrote the manuscript. WD approved the final version. All authors read and approved the final manuscript.

\section{Acknowledgments}

This work was supported by the National Natural Scientific Foundation of China (81070620, 30973477), the Natural Science Foundation of Jiangsu Province (No. BK2010590), the Medical Key Talented Person Foundation of Jiangsu Provincial Developing Health Project (RC2011080) and the School Fund of Nanjing Medical University (06NMUM013).

\section{Author details \\ 'Department of Biochemistry and Molecular Biology, Nanjing Medical University, Nanjing, People's Republic of China. ²Department of Oncology, First Affiliated Hospital, Nanjing Medical University, Nanjing, People's Republic of China. ${ }^{3}$ School of Life Science, Nanjing University, Nanjing, People's Republic of China. ${ }^{4}$ Department of Oncology, Second Affiliated Hospital, Nanjing Medical University, Nanjing, People's Republic of China. ${ }^{5}$ Department of Oncology, Affiliated Nanjing Hospital, Nanjing Medical University, Nanjing, People's Republic of China.}

Received: 18 January 2012 Accepted: 3 July 2012

Published: 9 August 2012

\section{References}

1. Jemal A, Siegel R, Xu J, Ward E: Cancer statistics, 2010. CA Cancer J Clin 2010, 60(5):277-300.

2. Verdecchia A, Francisci S, Brenner H, Gatta G, Micheli A, Mangone L, Kunkler I: Recent cancer survival in Europe: a 2000-02 period analysis of EUROCARE-4 data. Lancet Oncol 2007, 8(9):784-796.

3. Farazi TA, Spitzer Jl, Morozov P, Tuschl T: miRNAs in human cancer. J Pathol 2011, 223(2):102-115.

4. Bartel DP: MicroRNAs: genomics, biogenesis, mechanism, and function. Cell 2004, 116(2):281-297.

5. Chitwood DH, Timmermans MC: Small RNAs are on the move. Nature 2010, 467(7314):415-419

6. Kosik KS: MicroRNAs and cellular phenotypy. Cell 2010, 143(1):21-26.

7. Calin GA, Croce CM: MicroRNA signatures in human cancers. Nat Rev Cancer 2006, 6(11):857-866.

8. Ding J, Huang S, Wu S, Zhao Y, Liang L, Yan M, Ge C, Yao J, Chen T, Wan D, et al: Gain of miR-151 on chromosome 8q24.3 facilitates tumour cell migration and spreading through downregulating RhoGDIA. Nat Cell Biol 2010, 12(4):390-399.

9. Hui AB, Shi W, Boutros PC, Miller N, Pintilie M, Fyles T, McCready D, Wong D, Gerster K, Waldron L, et al: Robust global micro-RNA profiling with formalin-fixed paraffin-embedded breast cancer tissues. Lab Invest 2009, 89(5):597-606.

10. Wang $Y X$, Zhang XY, Zhang BF, Yang CQ, Chen XM, Gao HJ: Initial study of microRNA expression profiles of colonic cancer without lymph node metastasis. J Dig Dis 2010, 11(1):50-54.

11. Sun M, Liu XH, Li JH, Yang JS, Zhang EB, Yin DD, Liu ZL, Zhou J, Ding Y, Li SQ, et al: MiR-196a Is Upregulated in Gastric Cancer and Promotes Cell Proliferation by Downregulating p27kip1. Mol Cancer Ther 2012, 11(4):842-852

12. Wang $R$, Wang ZX, Yang JS, Pan X, De W, Chen LB: MicroRNA-451 functions as a tumor suppressor in human non-small cell lung cancer by targeting ras-related protein 14 (RAB14). Oncogene 2011, 30(23):2644-2658.

13. Braig S, Mueller DW, Rothhammer T, Bosserhoff A-K: MicroRNA miR-196a is a central regulator of HOX-B7 and BMP4 expression in malignant melanoma. Cellular and Molecular Life Sciences 2010, 67(20):3535-3548.

14. Mueller DW, Bosserhoff AK: MicroRNA miR-196a controls melanomaassociated genes by regulating HOX-C8 expression. Int J Cancer 2011, 129(5):1064-1074.
15. Guan Y, Mizoguchi M, Yoshimoto K, Hata N, Shono T, Suzuki SO, Araki Y, Kuga D, Nakamizo A, Amano T, et al: MiRNA-196 Is Upregulated in Glioblastoma But Not in Anaplastic Astrocytoma and Has Prognostic Significance. Clinical Cancer Research 2010, 16(16):4289-4297.

16. Luthra R, Singh RR, Luthra MG, Li YX, Hannah C, Romans AM, Barkoh BA, Chen SS, Ensor J, Maru DM, et al: MicroRNA-196a targets annexin A1: a microRNA-mediated mechanism of annexin A1 downregulation in cancers. Oncogene 2008, 27(52):6667-6678.

17. Schimanski CC, Frerichs K, Rahman F, Berger M, Lang H, Galle PR, Moehler M, Gockel I: High miR-196a levels promote the oncogenic phenotype of colorectal cancer cells. World J Gastroenterol 2009, 15(17):2089-2096.

18. Zhang SZ: Knockdown of c-Met by adenovirus-delivered small interfering RNA inhibits hepatocellular carcinoma growth in vitro and in vivo. Molecular Cancer Therapeutics 2005, 4(10):1577-1584.

19. Abramovich C, Humphries RK: Hox regulation of normal and leukemic hematopoietic stem cells. Curr Opin Hematol 2005, 12(3):210-216.

20. limura $T$, Pourquie $O$ : Hox genes in time and space during vertebrate body formation. Dev Growth Differ 2007, 49(4):265-275.

21. Moens CB, Selleri L: Hox cofactors in vertebrate development. Dev Biol 2006, 291(2):193-206.

22. Magli MC, Barba P, Celetti A, De Vita G, Cillo C, Boncinelli E: Coordinate regulation of HOX genes in human hematopoietic cells. Proc Natl Acad Sci U S A 1991, 88(14):6348-6352.

23. Daniels TR, Neacato II, Rodriguez JA, Pandha HS, Morgan R, Penichet ML: Disruption of HOX activity leads to cell death that can be enhanced by the interference of iron uptake in malignant B cells. Leukemia 2010, 24(9):1555-1565

24. Morgan R, Pirard PM, Shears L, Sohal J, Pettengell R, Pandha HS: Antagonism of HOX/PBX dimer formation blocks the in vivo proliferation of melanoma. Cancer Res 2007, 67(12):5806-5813.

25. Morgan R, Plowright L, Harrington KJ, Michael A, Pandha HS: Targeting HOX and PBX transcription factors in ovarian cancer. BMC Cancer 2010, 10:89.

26. Plowright $L$, Harrington $\mathrm{K}$, Pandha HS, Morgan R: HOX transcription factors are potential therapeutic targets in non-small-cell lung cancer (targeting HOX genes in lung cancer). Br J Cancer 2009, 100(3):470-475.

27. Shears L, Plowright L, Harrington K, Pandha HS, Morgan R: Disrupting the interaction between HOX and PBX causes necrotic and apoptotic cell death in the renal cancer lines CaKi-2 and 769-P. J Urol 2008, 180(5):2196-2201.

28. Aulisa L, Forraz N, McGuckin C, Hartgerink JD: Inhibition of cancer cell proliferation by designed peptide amphiphiles. Acta Biomater 2009, 5(3):842-853.

29. Mandeville I, Aubin J, LeBlanc M, Lalancette-Hebert M, Janelle MF, Tremblay $\mathrm{GM}$, Jeannotte $L$ : Impact of the loss of Hoxa5 function on lung alveogenesis. Am J Pathol 2006, 169(4):1312-1327.

30. Packer Al, Mailutha KG, Ambrozewicz LA, Wolgemuth DJ: Regulation of the Hoxa4 and Hoxa5 genes in the embryonic mouse lung by retinoic acid and TGFbeta1: implications for lung development and patterning. Dev Dyn 2000, 217(1):62-74.

31. Golpon HA, Geraci MW, Moore MD, Miller HL, Miller GJ, Tuder RM, Voelkel NF: HOX genes in human lung: altered expression in primary pulmonary hypertension and emphysema. Am J Pathol 2001, 158(3):955-966.

32. Raman V, Martensen SA, Reisman D, Evron E, Odenwald WF, Jaffee E, Marks J, Sukumar S: Compromised HOXA5 function can limit p53 expression in human breast tumours. Nature 2000, 405(6789):974-978.

33. Gendronneau G, Lemieux M, Morneau M, Paradis J, Tetu B, Frenette N, Aubin J, Jeannotte L: Influence of Hoxa5 on p53 tumorigenic outcome in mice. Am J Pathol 2010, 176(2):995-1005.

34. Raman V, Tamori A, Vali M, Zeller K, Korz D, Sukumar S: HOXA5 regulates expression of the progesterone receptor. J Biol Chem 2000, 275(34):26551-26555.

35. Chen $H$, Zhang $H$, Lee J, Liang $X$, Wu X, Zhu T, Lo PK, Zhang X, Sukumar S: HOXA5 acts directly downstream of retinoic acid receptor beta and contributes to retinoic acid-induced apoptosis and growth inhibition. Cancer Res 2007, 67(17):8007-8013.

doi:10.1186/1471-2407-12-348

Cite this article as: Liu et al:: MicroRNA-196a promotes non-small cell lung cancer cell proliferation and invasion through targeting HOXA5. BMC Cancer 2012 12:348. 